\title{
A DESIGN SUPPORT TOOL FOR IMPROVING MAINTENANCE SCHEDULING PROCESS
}

\author{
W. Khalid ${ }^{凶}$, I. Soleymani, K. V. Sigsgaard, K. B. Hansen, J. K. Agergaard and \\ N. H. Mortensen \\ DTU-Technical University of Denmark, Denmark \\ $\bigotimes$ wakha@mek.dtu.dk
}

\begin{abstract}
The challenge of user requirements for maintenance scheduling design in large asset-intensive industries suffers from lack of academic and empirical studies. Therefore, using a representative case study, this paper aims to: (1) identify the current practices and complex scheduling requirements; (2) propose a design support tool to optimize the maintenance scheduling process; and (3) report the gained benefits. The results reveal that the proposed tool can decrease the resource requirements, increase the capacity utilization, and reduce the cost while addressing the complex user requirements.
\end{abstract}

Keywords: design tools, design process, complex systems, maintenance, scheduling

\section{Introduction}

Pal (2014) stated that design can be considered as a decision-making process, meaning that if we have a problem then we need to develop a solution. To put it in another way, the design is the formulation of a plan to satisfy specific needs and to make something that has a physical reality (Pal, 2014). Usually, the output of the design can be a product, service (Sakao and Lindahl, 2009), process (Erwin, 2002), or computer software (Zhu, 2005) that must fulfill a user(s) requirement(s). However, the design output can also be a plan, translating a physical reality that must fulfill a user requirement (Márquez, 2002). An example of such an output is a maintenance schedule of a production facility that depicts when particular maintenance activities are to be carried out to fulfill the user requirements.

Maintenance is usually considered as cost-centered as it consumes a lot of operational costs (AlNajjar, 2012). However, the importance of the maintenance cannot be ignored as it is crucial for keeping the availability of the production equipment and is directly linked with the quality of the product itself and the safety of the equipment (Wang et al., 2007). If a company does not invest in the maintenance then non-maintenance related costs start to increase, hence it needs to find a balance between the two (Javier et al., 2014).

Tombari (1982) puts forth the importance of a "Maintenance Management System (MMS)" in industries and highlighted that a maintenance program can be considered as a sub-part of the whole production process, where the focus is to find the optimum use of available personnel, equipment, and facilities, and to keep an optimum balance between maintenance costs and costs of lost production (Tombari, 1982). Maintenance scheduling is a crucial part of the MMS to distribute the work and fulfill the capacity constraints, therefore, work can be executed without compromising any risks. This may look quite simple but can become quite tedious and complex in maintenance intensive industries. Many researchers 
have tried to address this issue. Some scholars such as Ben-Daya et al. (2009) have proposed some generic methods such as Gantt Charts, Critical Path Method (CPM), and Program Evaluation Review Techniques (PERT) but these methods are not sufficient to address the large complexities that exist in a design of maintenance schedule in large industries. Other researchers proposed more advanced mathematical programming and meta-heuristic approaches for optimization of the maintenance schedule (Roberts and Escudero, 1983; Duffuaa and Al-Sultan, 1997; Foong et al., 2005). However, these approaches lack the large scope of constraints and dependencies that exist in large maintenance intensive industries. Some researchers have proposed mathematical programming models for maintenance schedules within the oil \& gas sector. For example, Roberts and Escudero (1983) used an integer programming model to minimize the workers idle time while allocating workers with specific skills to maintenance tasks with known time intervals. Duffuaa and Al-Sultan (1997) proposed integer and stochastic programming approaches for maintenance scheduling. Their objective was to minimize the lateness of maintenance jobs and increase resource utilization while the priority of the jobs, skills required, and capacity are known under the uncertainty of incoming new maintenance jobs. Other researchers have proposed mathematical programming approaches for other sectors, for example, Foong et al. (2005) used Artificial Intelligence (AI) based heuristic method called Ant Colony Optimization (ACO) for optimizing the power plant maintenance schedule. The aim was to minimize cost or maximize reliability subject to load constraint, resource constraint, and window of time during which the maintenance can be carried out. Kovács et al. (2012) proposed a dynamic maintenance-scheduling model for wind farms in a dynamic environment that takes into account maintenance tasks, maintenance personnel, spare parts, weather conditions, task compatibility, and opportunity window, whereas, tries to minimize the production loss of the wind turbines. The literature in the maintenance scheduling and resource levelling is quite broad and Table 1 below highlights few of the works in this area.

Table 1. Brief literature on maintenance scheduling

\begin{tabular}{|l|l|l|}
\hline Sr. & Reference & Description \\
\hline 1 & (Denysiuk et al., 2017) & $\begin{array}{l}\text { A multi-objective optimization model for optimizing maintenance schedules for } \\
\text { pavements }\end{array}$ \\
\hline 2 & (Hadjaissa et al., 2016) & $\begin{array}{l}\text { Methods to improve quality of energy production and reducing costs. Methods } \\
\text { include mono-objective (make span), bi-objective (make span and training time) } \\
\text { and modified genetic algorithm }\end{array}$ \\
\hline 3 & (Verma et al., 2012) & $\begin{array}{l}\text { A non-dominated sorting genetic algorithm (NSGA-2) based multi-objective } \\
\text { optimization approach for optimizing maintenance scheduling of machinery on } \\
\text { ships }\end{array}$ \\
\hline 4 & (Worrall and Mert, 1980) & $\begin{array}{l}\text { Dynamic decision rules for day-to-day scheduling in oil and gas sector, method to } \\
\text { control backlog, and forecasting future work load }\end{array}$ \\
\hline 5 & (Nurcahyo et al., 2019) & Identification of key process areas in maintenance planning \\
\hline 7 & (Jiu et al., 2013) & $\begin{array}{l}\text { Minimization of weighted sum of aversion of start time of maintenance, change } \\
\text { over time, and train waiting time using mixed integer linear programming and } \\
\text { hybrid genetic algorithm in coal industry. }\end{array}$ \\
\hline 8 & $\begin{array}{l}\text { (Bartholomew-Biggs } \\
\text { et al., 2006) }\end{array}$ & $\begin{array}{l}\text { Dimensional reduction technique of maintenance scheduling for determining } \\
\text { optimal maintenance intervals }\end{array}$ \\
\hline 9 & $\begin{array}{l}\text { (Masmoudi and Haï, } \\
\text { Wa11) }\end{array}$ & $\begin{array}{l}\text { Genetic algorithm based heuristic approach of resource levelling to optimize } \\
\text { helicopter maintenance activity }\end{array}$ \\
\hline 10 & $\begin{array}{l}\text { (Stremel and Jenkins, } \\
1981)\end{array}$ & $\begin{array}{l}\text { Methods to incorporate and level the risk of uncertain weather outcomes in } \\
\text { maintenance schedules }\end{array}$ \\
\hline 11 & $\begin{array}{l}\text { (Cheung and Hui, 2002) } \\
\text { Mixed integer linear programming model for determining maintenance schedules } \\
\text { for both production and utility in order to minimize production loss. }\end{array}$ \\
\hline
\end{tabular}

Despite a long list of research work in the maintenance scheduling approaches, there is a lack of a comprehensive study that addresses the vast scope of the complex constraints in the maintenance 
scheduling design, especially at the long and medium time horizon level. Therefore, there exists a research gap in addressing the large scope of constraints that exist in designing of a maintenance schedule. Hence, it is very important to critically analyze the current practices in large asset-intensive industries and find ways to improve the design process of a maintenance schedule. Therefore, this paper will use a case study, representative of a very large asset-intensive industry and will try to fill the research gap. This leads us to the research questions (RQs) that this paper intends to answer:

1. What are the common challenges in an asset-intensive engineering company in designing a maintenance schedule?

2. How can the design process of a maintenance schedule be improved?

3. What are the obtained benefits of improving the design process of a maintenance schedule?

The paper is structured as follows; section 2 briefly describes the research methodology, section 3 introduces the case company and answers RQ1, section 4 presents the proposed design support tool and answers RQ2, section 5 provides a brief discussion and answers RQ3, lastly, conclusion and future work have been described.

\section{Research methodology}

The paper uses a case study approach proposed by Yin (1994) as it uses specific observations to draw generic conclusions. The rationale for using the case study approach is that the study is conducted in a case company and is the best way to elaborate the research. Moreover, as both qualitative and quantitative data are used in the paper for the analysis, hence it also follows mixed research methods research methodology (Creswell and Creswell, 2018).

The research comprises of a period of more than a year. The research started with the study of the internal documents of the case company and literature review. The study includes on average 2 to 3 visits per month of the case company. During the visits, meetings and semi-structured interviews were conducted with the planners, schedulers, and maintenance staff in the case company. The purpose of the visits were to understand the underlying phenomenon and constraints during the maintenance scheduling process to incorporate these into the model. Moreover, during each visit, results of the model were showed to the concerned persons and their feedbacks were used to modify the model until a satisfactory model was developed.

The literature review was conducted by using DTUFindit. Search words are comprised of but not limited to the followings: optimization of maintenance scheduling, maintenance scheduling in oil and gas, optimizing maintenance scheduling, maintenance resource levelling, maintenance scheduling, improving maintenance, maintenance management, and capacity levelling.

\section{Case company}

The case company is a large multi-national oil \& gas company and one of the major 7 oil companies in the world. The case company covers the entire supply chain of oil \& gas including upstream and downstream operations. It has more than 100,000 employees all over the world. The scope of this paper only covers the Danish part of the North Sea. The paper will use this case company and will first try to highlight the current maintenance scheduling practices. After this, the paper will try to highlight the common challenges in designing a maintenance schedule in the case company. Lastly, a design support tool will be proposed and the results of the implementation of this tool will be presented, highlighting the obtained benefits.

\subsection{Current maintenance management at the case company}

The case company has a complex maintenance management system. Figure 1 represents an overview of the maintenance management at the case company. The company has a maintenance management system, which is called the "Maintenance Work Management (MWM)" system. The MWM system is an integral part of the Enterprise Resource Planning (ERP) system also called the SAP system at the company, where all of the departments such as production, maintenance, planning, projects, finance, etc. are connected. The inputs from these departments are directly used in the MWM system and vice versa. This SAP system module responsible for managing maintenance is termed as a Computerized Maintenance Management System (CMMS) in the case company. 


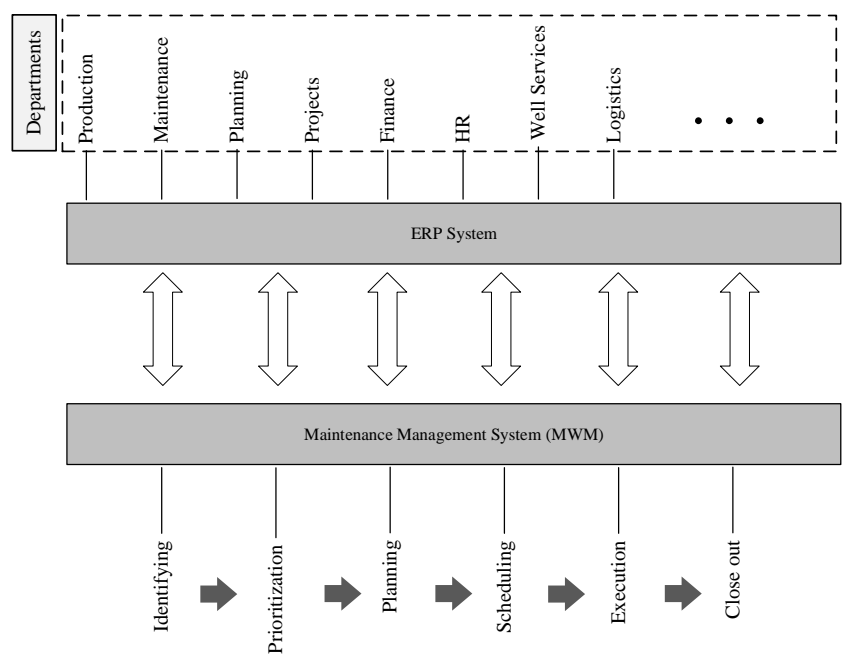

Figure 1. Maintenance management at the case company

The MWM system is comprised of six steps.

- In the first step, maintenance work is identified.

- As a second step, based on risk and likelihood, the work is given a certain priority defining a period in which the work should be carried out and finished. Once the priority is given, it does not change.

- The third step includes planning of maintenance work, which comprises defining the number of hours required, spare parts required, tools required, and the number of people required, etc.

- In the fourth step, the maintenance work is scheduled defining the time when it needs to be carried out.

- In the fifth step, the work is finally carried out as per the plan and schedule.

- Lastly, in the sixth step, the work is closed after the technical, quality, and financial approvals.

The scope of this paper is focused on the scheduling part of the MWM system.

\subsection{Current practices in designing a maintenance schedule}

The current scheduling practices at the case company comprise three main capacity leveling exercises; long term, medium term, and short term capacity leveling exercises and as a result of this aggregate, initial, and final maintenance schedules are generated. The capacity leveling is referred to as the leveling of required work against the available capacity over time.

Figure 2 represents an overview of the scheduling process at the case company. Maintenance works are being dumped regularly to be scheduled over time-ahead. Less priority works have more allowable time to be executed and finished, whereas, high priority works have less allowable time to be executed and finished. Preventive maintenance works (maintenance work of repetitive nature) are pre-planned and are called-in either automatically or manually to the MWM system, and are usually of less priority in nature. On the other hand, corrective maintenance works are usually of high priority. However, this is not always the case. Maintenance works that have a starting date between 90-120th day from day 0 are called longterm maintenance works. As per the standard operating procedures (SOPs), the schedulers are required to run a long-term capacity leveling exercise for these works so that the amount of work can be leveled against the available capacity and any extra capacity requirements can be evaluated. The maintenance works that have a starting date between 28-89th day from day 0 are called medium-term maintenance works. According to the SOP, schedulers are also required to run another capacity leveling exercise, which is called medium-term capacity leveling. In this exercise, work is leveled against the capacity over time with more precision and accuracy as information regarding material availability and capacities start to become more definite. The maintenance works that have starting dates between 7-27th day from day 0 are termed as short-term maintenance works. At this stage, final capacity leveling exercise takes place as at this point available capacity and material information become clear. Hence, the maintenance schedule is designed. After this, the schedule is locked while still allowing room for emergency break-in works with 
the highest priority. This is usually done by leaving almost $20 \%$ of the available capacity free for such works. Then in the next stage, the execution of the maintenance works starts and if any work cannot be executed for some reason, then, it is delayed and marked as backlog and reverted to the previous capacity leveling stages based on the new proposed starting date.

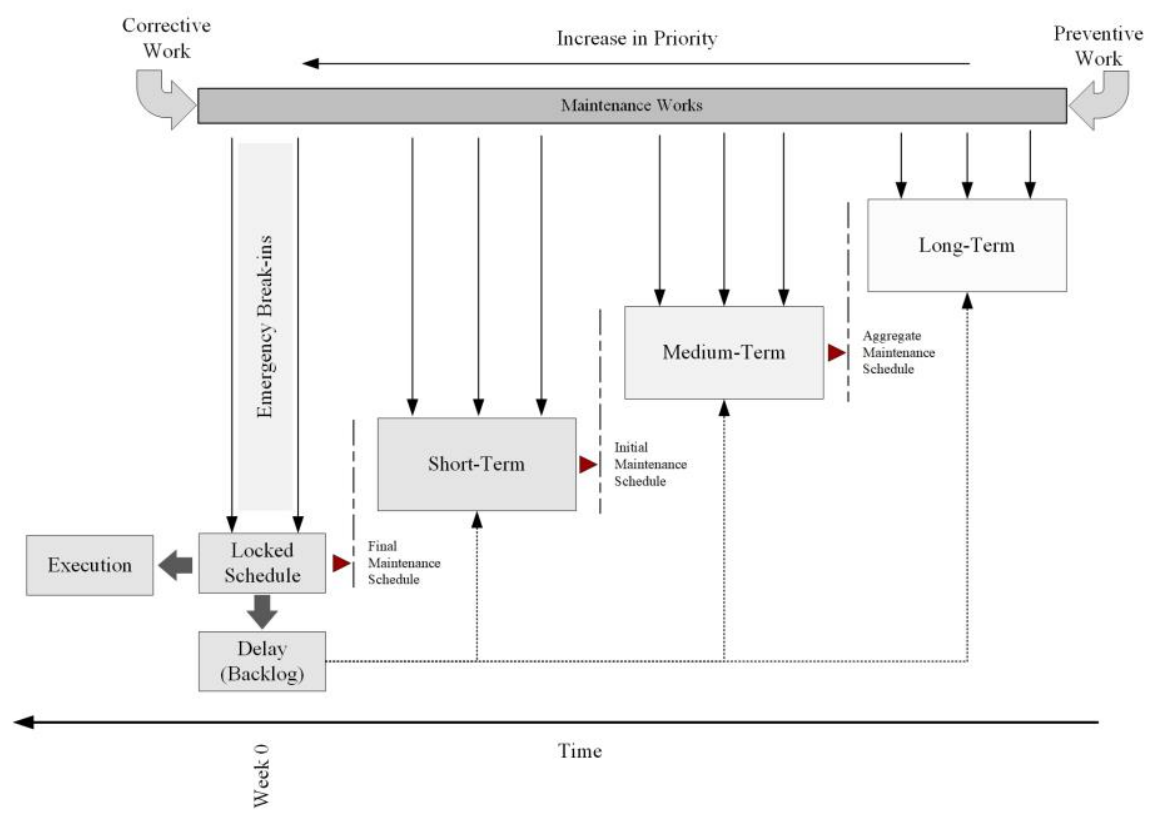

Figure 2. Current maintenance scheduling process at the case company

However, as per the interviews and visits to the case company, it has been found that the current practices do not include the capacity leveling for the medium and long term maintenance works and only final capacity leveling exercise is carried out. This is not desired as it puts extra pressure on the schedulers to firefight the work overload, especially in a high asset-intensive environment. Moreover, it results in bad compliance with maintenance schedules and a high number of backlogs. The schedulers use scheduling software like Primavera P6 as a supporting tool while designing the maintenance schedule. These tools are not designed to incorporate very high volumes of maintenance jobs and do not incorporate the high complexity of maintenance scheduling constraints.

\subsection{Challenges in designing a maintenance schedule}

There are some challenges in designing a maintenance schedule, which either has been noted down during the interviews or has been observed from analyzing the maintenance data. Firstly, there are many constraints and requirements that need to be fulfilled that makes it complex to design a maintenance schedule. A few of these requirements are mentioned in Figure 3.

\begin{tabular}{|c|c|c|c|}
\hline $\begin{array}{l}\text { a) Management of } \\
\text { resource allocation } \\
\text { (limited to maximum } \\
\text { capacity) }\end{array}$ & 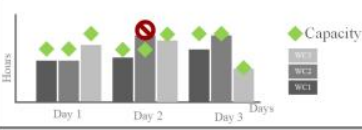 & $\begin{array}{l}\text { e) Management of start } \\
\text { date }\end{array}$ & $\diamond$ EASD $\quad$ Work 2 \\
\hline $\begin{array}{l}\text { b) Management of } \\
\text { offshore resources on } \\
\text { board (limited to } \\
\text { maximum capacity) }\end{array}$ & $Q_{\text {Allowed }}^{\text {Not }} \mathrm{POB}$ & $\begin{array}{l}\text { f) Management of } \\
\text { material }\end{array}$ & r. $\rightarrow$ 用 \\
\hline $\begin{array}{l}\text { c) Management of risk } \\
\text { for delaying the } \\
\text { maintenance work }\end{array}$ & Work 1-Prionity 2 & $\begin{array}{l}\text { g) Grouping of similar } \\
\text { works }\end{array}$ & 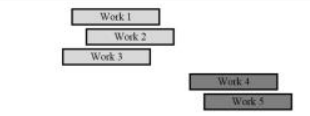 \\
\hline $\begin{array}{l}\text { d) Management of } \\
\text { dependencies among } \\
\text { operations }\end{array}$ & 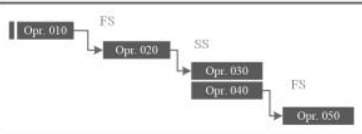 & \multicolumn{2}{|c|}{ 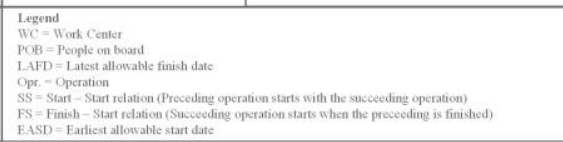 } \\
\hline
\end{tabular}

Figure 3. Requirements for designing the maintenance schedule in the case company 
A brief explanation of these requirements is given below:

a) Companies have some pools of capacity comprised of skilled workers, which termed work centers (WC). The capacity is in the maximum number of hours a WC can work in one day. For example, if there is a WC comprising of 10 mechanical technicians and each technician can work 8 hours per day then the capacity of that WC will be 80 hours. While designing a maintenance schedule this capacity level mustn't be exceeded.

b) Some special companies such as offshore oil \& gas companies have an additional constraint i.e. the maximum number of people that can be accommodated in an offshore platform. It is termed as the maximum people on board (POB). This limit can also not be exceeded.

c) While designing a maintenance schedule, it is important to manage the risk associated with delaying work. There is an allowable date (latest allowable finish date (LAFD)) until when work should be completed. However, in the case of limited capacity, some works can be delayed but in that case, it is important to delay a less important work rather than a highly important work.

d) Sometimes works have dependencies among them, for example, operation 1 should start along with the operation 2. This is termed as Start-Start (SS) relationship. Another example of the relationship is the Finish-Start (FS) relationship, which means that operation 2 can only start once the operation 1 is finished.

e) Sometimes works have a constraint on the date before that an operation cannot start. This is termed as the earliest allowable start date (EASD).

f) Some works have material requirements, which should also be considered while designing a maintenance schedule.

g) It is recommended to group similar works while designing a maintenance schedule as it will lead to less downtime and less usage of resources.

Hence, this section shows that there are quite some challenges in designing a maintenance schedule and mentions some of these challenges to answer RQ1.

\section{Findings}

In the previous sections, current practices and challenges in designing a maintenance schedule were highlighted. In this section, it will be analyzed that how designing the maintenance schedule can be improved and what the associated benefits are.

\subsection{Proposed design support tool}

As mentioned before, despite the availability of several mathematical programming approaches, there is a research gap that addresses the vast variety of constraints that exist in offshore oil \& gas platforms while designing a maintenance schedule. This paper proposes a design support tool that can perform optimum maintenance scheduling while addressing all of the constraints mentioned in section 3.2. Figure 4 presents the concept of the design support tool. The tool will be used to produce aggregate and initial maintenance schedules in the long and mid-term horizons respectively. This will remove the extra pressure on the short-term horizon and will provide a better overview of the resource requirements.

The proposed design support tool uses a combination of computer programming and a mixedinteger mathematical programming optimization model, where the objective is to minimize the weighted lateness of the maintenance jobs. The weights are direct representations of the priorities of the jobs, thus weighted lateness means that in case of not sufficient capacity, the model will delay the less priority job instead of a high priority job. The model is subject to all of the constraints that are mentioned in Figure 3. Figure 5 shows how the design support tool functions. It uses a combination of both computer programming and mathematical optimization model to handle all of the constraints mentioned in section 3.3.

First, the model takes in all of the data that exists in scheduling horizon. Second, based on the type of the maintenance jobs, it adopts a different strategy. It keeps in place all of the jobs related to well services, 
except it excludes the ones in the past. By keeping in place, means that the basic start and finish dates remains the same and are used as EASD and LAFD respectively. It adopts the same strategy for construction jobs, except it also group these jobs in case of a grouping requirement. It groups the jobs that have same subnetwork numbers or denominator numbers. The model uses a different strategy for corrective and preventive maintenance jobs. It only includes the jobs that have LAFD in future. If a job does not require a material then it uses the EASD and LAFD as it is. However, if a job requires a material then it uses a material status to incorporate EASD and LAFD. If material status is very late, purchase requisition (PR) is not released, material is backload, or there is a request for quotation (RFQ) then it will exclude these jobs from the analysis until responsible staff does not provide a clear material status. If the material status is on time then it uses the material requirement date (MRD) as EASD and LAFD remains the same. If the material status is very early or in stock then it uses today as the EASD and LAFD remains the same. If material status is early then it uses minimum of the EASD and MRD as the EASD and LAFD remains the same. If material status is late then it uses maximum of EASD and MRD as the EASD and LAFD remains the same. Third, after this, the data is collected, transformed and extracted to feed into the mathematical optimization model. Figure 5 shows the model in the right column. The mathematical model is self-explanatory, where it calculates the start and finish dates of each job and operation while minimizing the weighted lateness. This is a proposed design support tool through which the designing of the maintenance schedule can be improved and thus answers the RQ2.

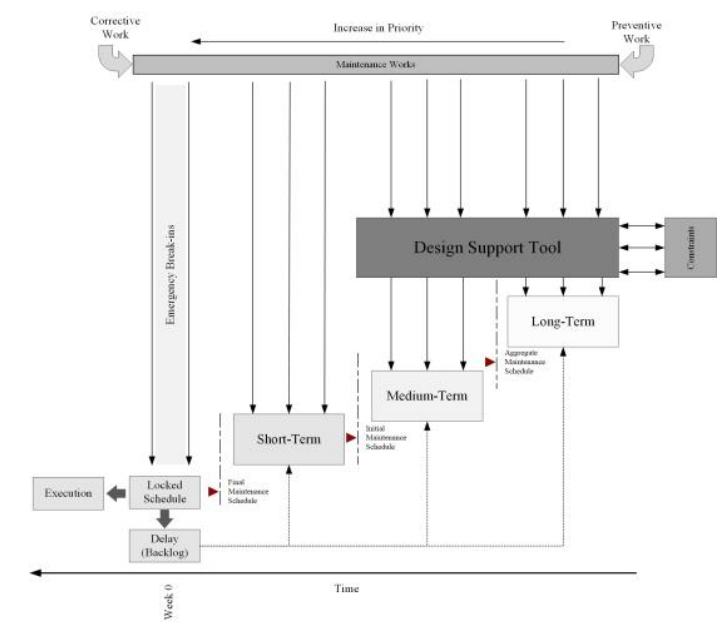

Figure 4. A proposed design support tool for designing a maintenance schedule

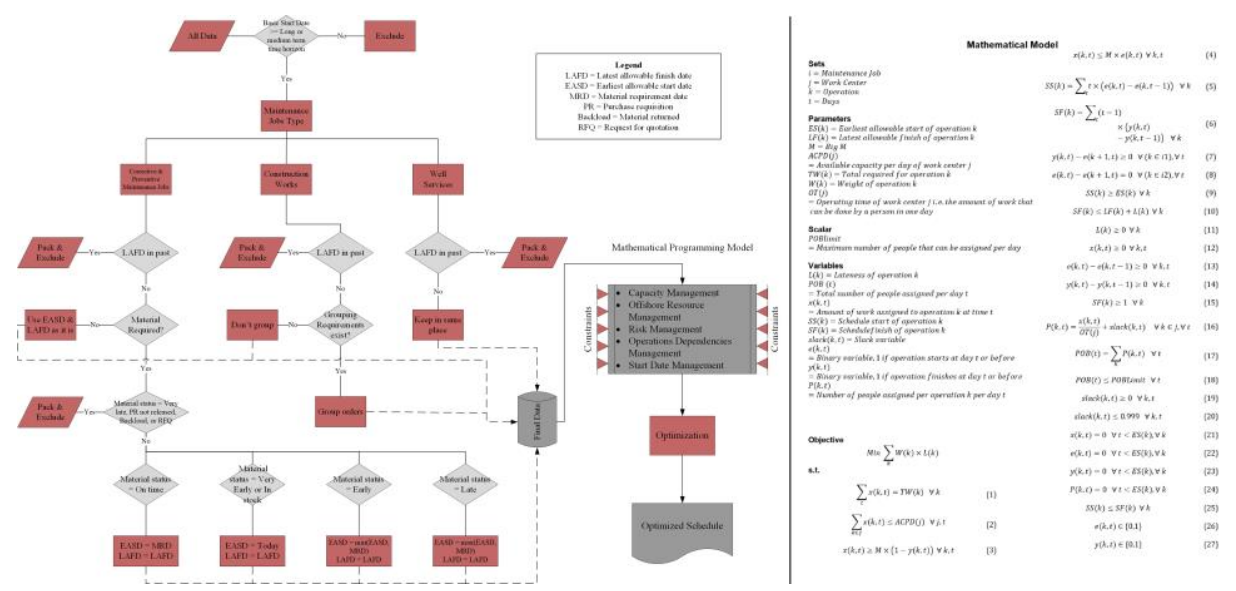

Figure 5. How design support tool functions

\subsection{Results of the proposed tool}

Figure 6 shows the results of the proposed design support tool before and after the implementation. The Figure 6a shows the distribution of the workload against time before and after the optimization. 
The Figure $6 \mathrm{~b}$ shows the capacity utilization of one of the WCs. It can be seen that the capacity utilization was more than $100 \%$ before the implementation of the tool in September 2019, which is physically impossible, as it is not allowed to allocate more capacity than available. The Figure $6 \mathrm{c}$ shows the number of people that are required at the offshore platform before and after the optimization. It can be seen that people's requirements have decreased significantly with the use of the proposed method. This is because the proposed tool by utilizing the available resources distributes the work in a way that requires fewer people to carry out the same amount of work.

The proposed tool was used for around 3 months (Jun 2019 - Aug 2019) in the case company for testing and verification. Once, the tool was verified then it took approximately 1 week to apply this in the case company for the specific case presented in Figure 6.

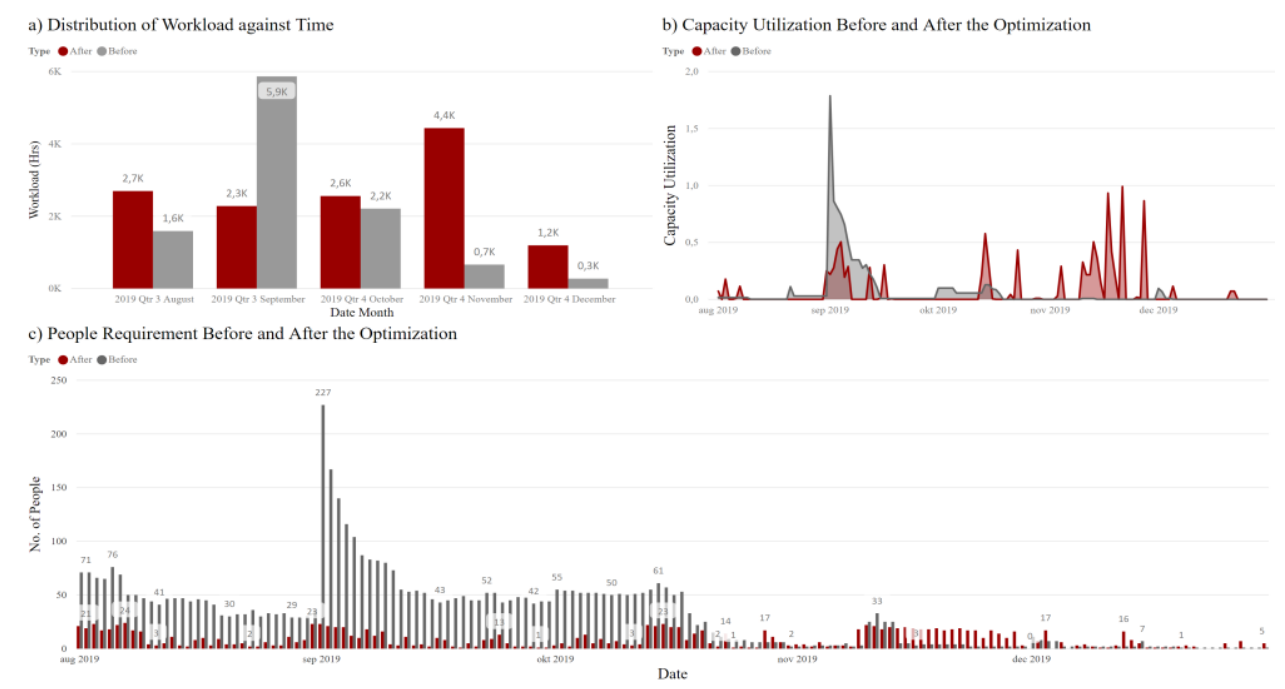

Figure 6. Results of the proposed optimization model

\section{Discussion}

The proposed tool was implemented for the long-term time horizon for making an aggregate maintenance schedule. The mathematical model within the proposed tool was run in an optimization software called GAMS. It had 1.14 million variables and 1.165 million equations and it took approximately 14 hours to solve the model. The model was solved on a computer with the specifications of Intel Xeon CPU 3.60 GHz with 6 Cores and Installed Physical Memory of 64 GB.

The results of the proposed model are shown in Figure 6. First, it can be seen that the number of people required to perform the required amount of work has been reduced significantly. This is one of the biggest benefits of utilizing the proposed model. As in an offshore oil \& gas platform, it is very expensive to accommodate and transport people to carry out the maintenance job. Hence, by reducing the number of people required, the associated cost can be reduced drastically.

Second, making a maintenance schedule at the aggregate level can help in estimating the people requirement very early on and thus can help the case company in managing the transport and accommodation of the people and managing third party contractors and vendors. The tool runs the algorithm in the background and requires minimum inputs from the schedulers. Hence, the tool will be used as a design support tool for the scheduler without overburdening him/her. Additionally, this will speed up the scheduling process and will help in the oncoming process steps.

Third, due to the better use of available resources, it will become easier to comply with the maintenance schedule without significant unwanted disruptions and the performance indicator for the maintenance schedule compliance will improve. As a consequence of this, there will be fewer delayed maintenance jobs and the number of backlogs will decrease gradually.

Fourth, currently, in the case company, the workers and contractors are planned and scheduled separately and thus, transported via helicopter to the oil \& gas platforms as per their planned shifts. Whereas, the work is then tried to be scheduled as per workers' shifts. This way of managing people is costly and results in a lot of idle time. Once the people's requirements are known beforehand then the 
contractors and workers can be called in for the required work as per the maintenance schedule and not the other way around. This is another advantage that this support tool can provide. The benefits mentioned in the above paragraphs answer RQ3.

The whole process involves several interviews and company visits, data extraction from SAP, data cleaning and transformation in R, optimization in GAMS, data post-processing and transformation in $\mathrm{R}$, and visualization in Power BI. Relevant stakeholders verified the results.

Previous works such as by Denysiuk et al. (2017), Hadjaissa et al. (2016), Verma et al. (2012), Masmoudi and Haït (2011), and Cheung and Hui (2002) are very limited to the specific equipment or focus on production or cost and do not take into account all maintenance jobs in MWM. Moreover, they do not incorporate such large number of constraints. Hence, the proposed model, built on the knowledge of mathematical modelling and computer algorithm, is a novel way of improving maintenance scheduling design process.

As the model pushes the less priority jobs to end up in backlog in case of not enough capacity, there might be an issue of recurrent deferrals of the same jobs in the next rounds, as it will have the same priority. However, increasing the priority of the deferred jobs can address this issue.

\section{Conclusion, limitations, and future works}

The purpose of this paper was to propose a design support tool for improving the maintenance scheduling process in an asset-intensive industry. The paper utilized a case study and aimed at answering the three RQs. The study started by highlighting the current practices at the case company. It was found that current practices are not optimal and there is a lack of design tool for long and medium-term capacity levelling in companies dealing with highly engineered maintenance services. Answering the RQ1, it was also found that there are quite several challenges that exist while designing a maintenance schedule. Capacity constraint, offshore resources accommodation, risk management, dependencies among operations, and material management are some of these constraints. The paper proposed a design support tool based on the principles of computer programming and mathematical programming approaches that can be used for optimized allocation of resources to the maintenance tasks and thus answered the RQ2. Finally, answering the RQ3, the paper highlighted the benefits of using the design support tool, which include reduced people requirement, increased capacity utilization, reduced cost, estimation of resource requirement at the aggregate level, reduction in backlog, reduced time in designing of the maintenance schedule, and people call-ins as per the maintenance schedule.

In addition to contributing to both the academic literature and managerial practice, the present study identifies some limitations that could be addressed in future research. The decision to conduct this study in a single company limits the generalizability of our results. In addition, the unique organizational context did not allow us to detect potential contingencies due to specific contexts, which could have affected the results. Therefore, in order to enhance the external validity of our results and to identify relevant contingencies, future qualitative and quantitative studies could replicate, confirm or augment the results of this study in different research settings or within larger or smaller statistical samples. Finally, we also focused only on companies with highly engineered maintenance services. The tool can be tested and verified on smaller companies with less complicated maintenance services.

\section{Acknowledgments}

The paper acknowledges the support and fund provided by the Danish Hydrocarbon Research \& Technology Center (DHRTC) for this study. Moreover, the authors also realize and appreciate the efforts put in by Sara Shafiee in reviewing this paper.

\section{References}

Al-Najjar, B. (2012), "Maintenance impact on company competitiveness and profit", In: Asset Management, Springer, Netherlands, pp. 115-141. https://doi.org/10.1007/978-94-007-2724-3

Bartholomew-Biggs, M., Christianson, B. and Zuo, M. (2006), "Optimizing preventive maintenance models", Computational Optimization and Applications, Vol. 35 No. 2, pp. 261-279. https://doi.org/10.1007/s10589006-6449-x

Ben-Daya, M. et al. (2009), Handbook of Maintenance Management and Engineering, Springer, London. https://doi.org/10.1016/978-1-84882-472-0 
Cheung, K.Y. and Hui, C.W. (2002), "Short-term site-wide maintenance scheduling", Computer Aided Chemical Engineering, Vol. 10 No. C, pp. 655-660. https://doi.org/10.1016/S1570-7946(02)80137-7

Creswell, J.W. and Creswell J.D. (2018), Research Design: Qualitative, Quantitative, and Mixed Method Approaches, Sage Publications, USA.

Denysiuk, R. et al. (2017), "Two-stage multiobjective optimization of maintenance scheduling for pavements", Journal of Infrastructure Systems, Vol. 23 No 3, https://doi.org/10.1061/(asce)is.1943-555x.0000355

Duffuaa, S. and Al-Sultan, K. (1997), "Mathematical Programming Approaches for the Management of Maintenance Planning and Scheduling", Journal of Quality in Maintenance Engineering, Vol. 3 No. 3, pp. 163-176. https://doi.org/10.1108/13552519710177943

Erwin, D.L. (2002), Industrial Chemical Process Design, McGraw-Hill.

Foong, W.K., Maier, H.R. and Simpson, A.R. (2005), "Ant Colony Optimization for Power Plant Maintenance Scheduling Optimization", Proceedings of the 7th Annual Workshop on Genetic and Evolutionary Computation, Washington DC, USA, June 25-29, 2005, ACM Press, pp. 354-357. https://doi.org/10.1145/1102256.1102335, https://doi.org/10. 1145/1102256

Hadjaissa, B. et al. (2016). "Bi-objective optimization of maintenance scheduling for power systems" International Journal of Advanced Manufacturing Technology, Vol. 85 No. 5-8, pp. 1361-1372. https://doi.org/10.1007/s00170-015-8053-7

Javier, S., Richard, W. and José, M.T. (2014), "Equipment Efficiency: Availability, Performance, and Maintenance", In: Improving Production with Lean Thinking, John Wiley \& Sons, New Jersey, pp. 96-119. https://doi.org/10. 1002/9781118984031.ch6

Jiu, S., Zhou, Z. and Liu, J. (2013), “The equipment maintenance scheduling problem in a coal production system", International Journal of Production Research, Vol. 51 No. 17, pp. 5309-5336. https://doi.org/10.1080/00207543. 2013.808773

Kovács, A. et al. (2012), "Scheduling the Maintenance of Wind Farms for Minimizing the Production Loss", IFAC Proceedings Volumes, Vol. 44 No. 1, pp. 14802-14807. https://doi.org/10.3182/20110828-6-IT-1002.02366

Márquez, A.C. (2002), "A Method to Design a Maintenance Plan", In: The Maintenance Management Framework, Springer, London, pp. 133-155. https://doi.org/10.1007/978-1-84628-821-0_11

Masmoudi, M. and Haït, A. (2011), "A GA-based fuzzy resource levelling optimization for helicopter maintenance activity", In: Proceedings of the 7th Conference of the European Society for Fuzzy Logic and Technology, Vol. 1 No. 1, pp.665-672.

Nurcahyo, R. et al. (2019), "Maintenance Planning Key Process Area: Case Study at Oil Gas Industry in Indonesia", In: IEEE International Conference on Industrial Engineering and Engineering Management, pp. 1704-1708. https://doi.org/10.1109/ieem.2018.8607527

Pal, S. (2014), "Basics of design process", In: Design of Artificial Human Joints \& Organs, Springer, US, pp. 41-49. https://doi.org/10.1007/978-1-4614-6255-2

Roberts, S.M. and Escudero, L.F. (1983), "Scheduling of Plant Maintenance Personnel”, Journal of Optimization Theory and Applications, Vol. 39 No. 3, pp. 323-343. https://doi.org/10.1007/BF00934541

Sakao, T. and Lindahl, M. (2009), Intoduction to Product/Service-System Design, Springer, London. https://doi.org/10. 1007/978-1-84882-909-1

Stremel, J.P., and Jenkins, R.T. (1981), "Maintenance scheduling under uncertainty", In: Ieee Transactions on Power Apparatus and Systems, Vol. 100 No. 2, pp. 460-465. https://doi.org/10.1109/TPAS.1981.316902

Tombari, H.A. (1982), "Designing a Maintenance Management System", Production and Inventory Management, Vol. 23 No. 4, pp. 139-147.

Verma, A.K. et al. (2012), "Optimization of maintenance scheduling of ship borne machinery for improved reliability and reduced cost”, International Journal of Reliability, Quality, and Safety Engineering, Vol. 19 No. 3. https://doi.org/10.1142/s0218539312500143

Wang, L., Chu, J. and Wu, J. (2007), "Selection of Optimum Maintenance Strategies Based on a Fuzzy Analytic hierarchy process", International Journal of Production Economics, Vol. 107 No. 1, pp. 151-163. https://doi.org/10.5267/j.msl.2014.3.028

Worrall, B.M. and Mert, B. (1980), "Application of dynamic scheduling rules in maintenance planning and scheduling", International Journal of Production Research, Vol. 18 No. 1, pp. 57-71. https://doi.org/10.1080/00207548008919649

Yin, R.K. (1994), Case Study Research: Design and Methods, Sage Publications.

Zhang, T., Nakamura, M. and Hatazaki, H. (2002), "Optimizing maintenance scheduling of equipment by element maintenance interval adjustment considering system availability”, In: Ieee Power Engineering Society Winter Meeting, Vol. 1-2, Conference Proceedings, pp. 205-210.

Zhu, H. (2005), Software Design Methodology, Elsevier. https://doi.org/10.1016/B978-0-7506-6075-4.X5000-6 